\title{
MODELAGEM MATEMÁTICA SEMI-MECANÍSTICA DA HIDRÓLISE ENZIMÁTICA DO BAGAÇO DE CANA-DE-AÇÚCAR
}

\author{
R. A.AKISUE ${ }^{1}$ e R.SOUSA $\mathrm{Jr}^{1}$ \\ ${ }^{1}$ Universidade Federal de São Carlos, Departamento de Engenharia Química \\ E-mail para contato: ruy@ufscar.br
}

\begin{abstract}
RESUMO - O etanol de segunda geração é uma alternativa para as 158,5 milhões de toneladas de bagaço de cana-de-açúcar produzidas anualmente pelo setor sucro-alcooleiro no Brasil, e pode ser obtido por duas rotas: termoquimica ou bioquímica (ácida ou enzimática). Na rota bioquímica enzimática, materiais lignocelulósicos (como o bagaço de cana) são hidrolisados em açúcares fermentescíveis. O modelo para reação catalítica enzimática heterogênea com contribuições dos efeitos fractal e/ou de "jamming" e o modelo HCH-1 são modelos cinéticos semi-mecanísticos que se apresentam como alternativas para aplicação no design, otimização e scale-up dos reatores enzimáticos. Neste trabalho, fazendo uso dométodo de otimização local clássico deLevenbergMarquardtpara ajuste de parâmetros (implementado em Fortran), foi possível ajustar os modelos citados a dados experimentais de hidrólise disponíveis em nosso laboratório.
\end{abstract}

\section{INTRODUÇÃO}

É crescente a pesquisa na área de produção de etanol de segunda geração. Embora ainda relativamente recente, ela mobiliza um grande número de pesquisadores em universidades e em programas como o Programa FAPESP de Pesquisa em Bioenergia (Bioen) que buscam tornar a produção de etanol de segunda geração, em escala industrial, economicamente viável (Marques, 2009). A diferença básica entre o etanol de primeira geração e o de segunda é que enquanto no primeiro caso o etanol é produzido através de uma extração e fermentação do caldo, o segundo é produzido através da hidrólise de materiais lignocelulósicos que posteriormente serão fermentados para a produção do etanol. (Buckeridgeet al., 2010).

Materiais lignocelulósicos podem ser processados por duas rotas distintas para a produção de bioetanol: uma rota termoquímica ou uma rota bioquímica. A primeira é baseada nos processos de pirólise e/ou gaseificação desses materiais, obtendo-se um gás de síntese que posteriormente passará por um processo catalítico adequado. Já a segunda rota (e a mais utilizada) é baseada nos processos de hidrólise e fermentação dos materiais lignocelulósicos, o que pode requerer um pré-tratamento do material. Basicamente, a hidrólise pode ser ácida ou enzimática (Loraet al., 2008).

Na hidrólise enzimática o agente hidrolisante são conjuntos de enzimas, chamadas celulases, que são misturas de endoglucanases e celobiohidrolases (GalbeeZacchi, 2007). Mas é necessário um 
pré-tratamento do material (Almeida, 2012). Além disso, este processo exige um maior tempo de residência (Ogedae Petri, 2010).

A literatura atual apresenta diversos modelos de hidrólise enzimática de materiais lignocelulósicos, alguns com o objetivo de aplicação destes modelos cinéticos no design, otimização de processos e scale-up da engenharia de biorreatores(Sousa Jret al., 2011). Devido a fatores como a complexidade do substrato, ação das enzimas e a interação enzima - substrato(Sousa Jret al., 2011), a modelagem do processo de hidrólise está longe do trivial. Uma alternativa interessante dentro da perspectiva de aplicação em engenharia de biorreatores são os modelos semi-mecanísticos. Alguns deles têm como base o modelo clássico (homogêneo) de Michaelis Mentental como o modelo HCH-1 estudado por O`Dwyer., et al(2007) e o modelo cinético para reação catalítica enzimática heterogênea com contribuições dos efeitos fractal e/ou de "Jamming” estudado por Xu e Ding (2007).

O modelo HCH1 é um dos possíveis modelos de Michaelis Menten modificado com inibição não competitiva. Observou-se que a taxa de hidrólise da celulose sofre um decréscimo, geralmente antes de todo o substrato ser consumido. Isto pode ser explicado por uma possível inibição pelo produto, baixa reatividade do substrato (devido à forma cristalina) ou à inativação e muitas vezes perda da enzima devido a uma adsorção irreversível com lignina (O`Dwyeret al., 2007). A inibição pelo produto da celulase é uma das limitações que pode restringir seu uso prático em processos de conversão de biomassa.

O modelo HCH-1 utiliza inibição não competitiva e pode ser descrito pela equação 1.

$$
V=-\frac{d G_{x}}{d t}=\frac{\kappa G_{x} E i}{\alpha+\phi G_{x}+\varepsilon E}
$$

Onde $G_{x}$ é a concentração de celulose, $E$ é a concentração de enzima, $\phi$ é a fração da superfície da celulose que esta livre para ser hidrolisada e $\kappa, \alpha$ e $\varepsilon$ são parâmetros que descrevem o grau de reatividade do substrato. A Equação 1 pode ser linearizada, obtendo-se a Equação 2.

$$
\frac{1}{V}=\frac{\alpha+\phi G_{x}}{\kappa G_{x} i} \frac{1}{E}+\frac{\varepsilon}{\kappa G_{x} i}
$$

Percebe-se que tanto o coeficiente linear quanto o coeficiente angular estão multiplicados por um fator $1 / \mathrm{i}$, o que é um indicativo que há um padrão de inibição não competitivo clássico (O`Dwyeret al., 2007).

O segundo modelo anteriormente citado é o modelo cinético para reação catalítica enzimática heterogênea com contribuições dos efeitos fractal e de "Jamming” estudado por Xu e Ding (2007). Aplicou-se um formalismo fractal geral à cinética de Michaelis Menten homogênea. Além disso, 
quantificou-se o bloqueio cinético causado por um excesso de enzima sobre o substrato, como pode ser visto na Equação 3 (Xu e Ding, 2007).

$$
\left(1-\frac{E}{j S}\right) \frac{k_{2} E t^{1-f}}{1-f}=P-k_{m} \ln \left(1-\frac{P}{S}\right)
$$

Onde E é a concentração de enzimas, $S$ é a concentração de substrato, j é o fator de "Jamming", f é a dimensão fractal e $\mathrm{k}_{\mathrm{m}}$ é a constante de Michaelis Menten. A equação não integrada do modelo é representada pela Equação 4 (Xu e Ding, 2007).

$$
\frac{d[P]}{d t}=\left(1-\frac{E}{j[S]}\right) k_{2} E t^{-f}\left(\frac{[S]}{k_{m}+[S]}\right)
$$

Quando f tende a zero, tem-se que o efeito fractal pode ser desprezado, obtendo-se a Equação 5.

$$
\frac{d[P]}{d t}=\left(1-\frac{E}{j[S]}\right) k_{2} E\left(\frac{[S]}{k_{m}+[S]}\right)
$$

Após o detalhamento teórico de alguns modelos, é importante discorrer sobre o método de otimização (ajuste de parâmetros).

Em métodos de otimização local, a busca iterativa começa em um ponto inicial e a região em torno da vizinhança deste ponto é examinada. Os principais métodos dessa classe são: algoritmo baseado em gradiente (gradiente descendente), Newton, Gauss Newton e Levenberg - Marquardt. Este último é uma combinação do algoritmo de Gauss e do gradiente descendente. Assim, ele dará resultados robustos, porém levando mais tempo para convergir. O método de Levenberg-Marquardt é aplicado na maioria dos problemas de ajuste paramétrico de modelos (Nelles, 2000).

\section{OBJETIVOS}

Diante do exposto, o presente trabalho tem como objetivo aplicar os modelos HCH-1 de Hidrólise Enzimática (O’Dwyeret al., 2007) e o Modelo de Catalise Enzimática Heterogênea com contribuição dos efeitos fractal e/ou de "Jamming" (Xu e Ding, 2007) aos dados de Carvalho(2011).

\section{METODOLOGIA}

Para o ajuste e análise dos modelos cinéticos, utilizaram-se os dados de hidrólise enzimática do bagaço de cana-de-açúcar fornecidos por Carvalho (2011) tal como mostra a tabela 1. 
Tabela 1: Valores de concentração de produto obtidas em função do tempo. À esquerda o conjunto de dados 1 e à direita o conjunto de dados 2. (Carvalho, 2011)

\begin{tabular}{|c|c|c|c|}
\hline Tempo (min) & $\begin{array}{c}\text { Concentração de } \\
\text { Produto (g/l) }\end{array}$ & Tempo (min) & $\begin{array}{c}\text { Concentração de } \\
\text { Produto (g/l) }\end{array}$ \\
\hline 0 & 0 & 0 & 0 \\
\hline 15 & 0,899 & 10 & 2,375 \\
\hline 30 & 2,203 & 20 & 2,964 \\
\hline 60 & 5,175 & 30 & 3,096 \\
\hline 120 & 9,418 & 60 & 3,628 \\
\hline 240 & 12,54 & 120 & 11,57 \\
\hline 420 & 19,75 & 240 & 23,99 \\
\hline 1740 & 32,53 & 540 & 36,73 \\
\hline 2760 & 31,96 & 1200 & 46,03 \\
\hline & & 1770 & 42,9 \\
\hline & & 3090 & 54,3 \\
\hline
\end{tabular}

Utilizaram-se os conjuntos de dados contidos na Tabela 1 acima e as condições iniciais $\mathrm{E}=$ $7,83 \mathrm{~g} / \mathrm{l}$ e $\mathrm{S} 0=33,33 \mathrm{~g} / \mathrm{l}$ para o primeiro conjunto de dados e $\mathrm{E}=13,05 \mathrm{~g} / \mathrm{l}$ e $\mathrm{S}_{0}=77,77 \mathrm{~g} / \mathrm{l}$ para o segundo conjunto de dados. Para aaplicação do método de ajuste de parâmetros de LevenbergMarquadt,os modelos cinéticos foram ajustados utilizando software in house in Fortran.

\section{RESULTADOS E DISCUSSÕES}

\subsection{Resultados do Ajuste do Modelo de Catálise Enzimática com Contribuição do Efeito de "jamming” de Xu eDing (2007).}

Os resultados obtidos para $\mathrm{k}_{\mathrm{m}}, \mathrm{V}_{\text {máx }}\left(=\mathrm{k}_{2} \mathrm{E}\right)$ e $\mathrm{o}$ parâmetro $\mathrm{j}$ ajustados $(\operatorname{com} \mathrm{f}=0)$ são mostrados na Tabela 2.

Tabela 2: Valores de $\mathrm{k}_{\mathrm{m}}, \mathrm{V}_{\text {máx }}$ e o parâmetro j ajustados para os 2 conjuntos de dados

\begin{tabular}{|l|l|l|l|} 
Conjunto de dados & $\mathrm{km}(\mathrm{g} / \mathrm{l})$ & $\mathrm{V}_{\text {máx }}(\mathrm{g} /(\mathrm{l} \cdot \mathrm{min}))$ & $\mathrm{j}$
\end{tabular}




\begin{tabular}{|l|l|l|l|}
\hline 1 & 17.57 & 0.094 & 8.54 \\
\hline 2 & 17.57 & 0.270 & 0.41 \\
\hline
\end{tabular}

Houve um aumento no valor de $\mathrm{V}_{\text {máx }}$ do conjunto 2 em relação ao conjunto 1 . Isto se deve ao fato de que $\mathrm{V}_{\text {máx }}$ é função apenas da constante $\mathrm{k}_{2}$ e da carga enzimática. Como esta aumentou 66,7\% em relação à carga do conjunto, o valor de $\mathrm{V}_{\text {máx }}$ também aumentou proporcionalmente (75\%). Pode se perceber também que houve uma diminuição significativa no parâmetro j, o que acarretou em uma diminuição na velocidade de hidrólise, ou seja, houve um aumento no efeito “jamming”. Isso ocorreupoiso aumento da concentração inicial de substrato e da carga enzimática fizeram com que mais produto fosse formado. Provavelmente, parte desse produto permaneceu adsorvido na matriz sólida, contribuindo para um maior efeito de "jamming" nos sítios ativos de adsorção no substrato sólido.

A Figura 1 mostra gráficos construídos no programa Origin para concentração de produto em relação ao tempo, considerando os dados experimentais e os valores obtidos nas simulações.
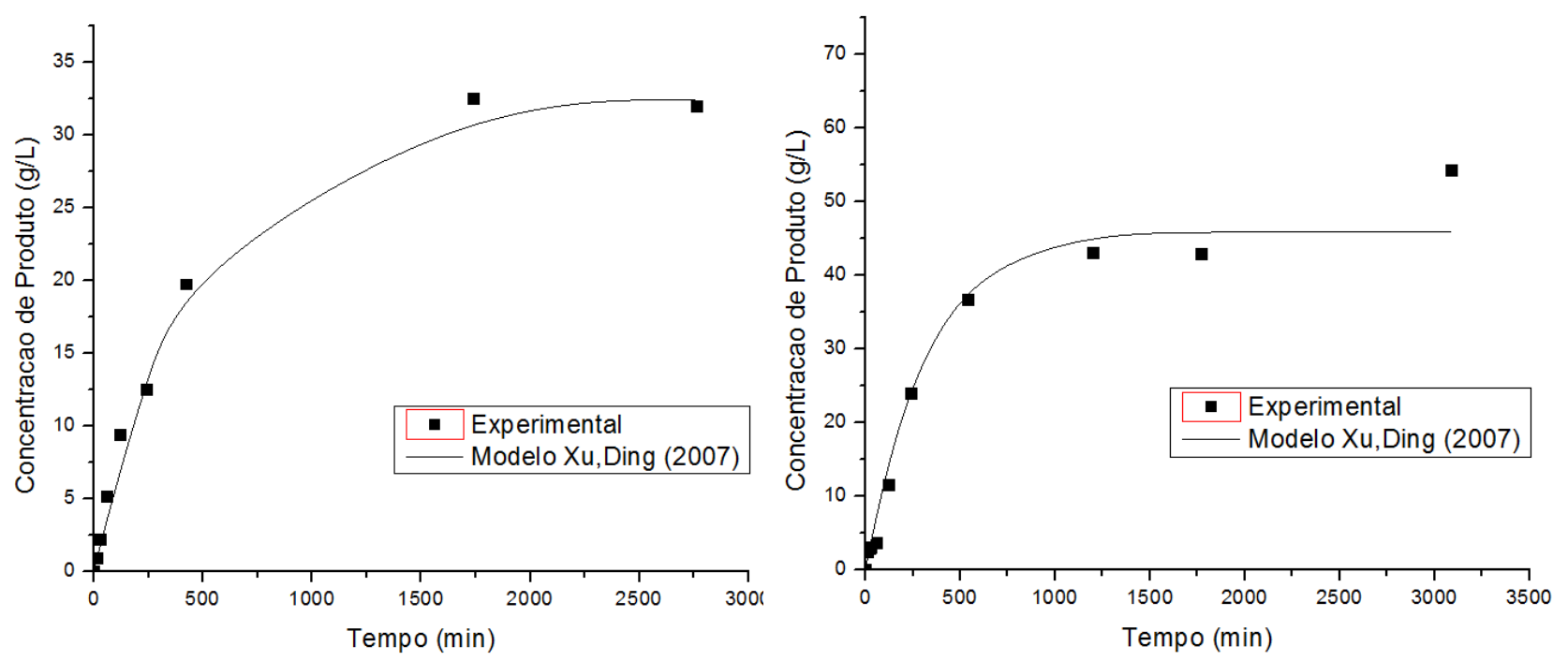

Figura 1Gráficos de concentração de produto em função do tempo. À esquerda o gráfico relacionado ao conjunto 1 e à direita o gráfico relacionado ao conjunto 2 .

Pode-se notar que em ambos os conjuntos há uma boa correspondência entre o modelo de Xu e Ding (2007) com efeito de "jamming" (linha contínua) e os dados experimentais (pontos), o que valida o modelo adotado.

\subsection{Resultados do Ajuste do Modelo HCH-1 de O’Dwyer, et al. (2007)}


Os resultados obtidos para $\mathrm{V}_{\text {máx }}^{`}(=\mathrm{kE}), \mathrm{i}, \alpha, \phi$ e $\varepsilon$ ajustados são mostrados na Tabela 3.

Tabela 3: Valores de $\mathrm{V}_{\text {máx }}$, i, $\alpha, \phi$ e $\varepsilon$ ajustados para os 2 conjuntos de dados

\begin{tabular}{|c|c|c|c|c|c|}
\hline Conjunto de dados & $\alpha(\mathrm{g} / \mathrm{l})$ & $\mathrm{V}_{\text {máx }}^{\prime}(\mathrm{g} /(\mathrm{l} \cdot \mathrm{min}))$ & $\mathrm{i}$ & $\varepsilon$ & $\phi$ \\
\hline 1 & 2,57 & 0,105 & 0,5 & 1,22 & 0,5 \\
\hline 2 & 2,57 & 0,175 & 0,18 & 1,56 & 0,65 \\
\hline
\end{tabular}

A Figura 2 mostra os gráficos gerados pelo programa Origin, que mostram a concentração de produto em relação ao tempo.
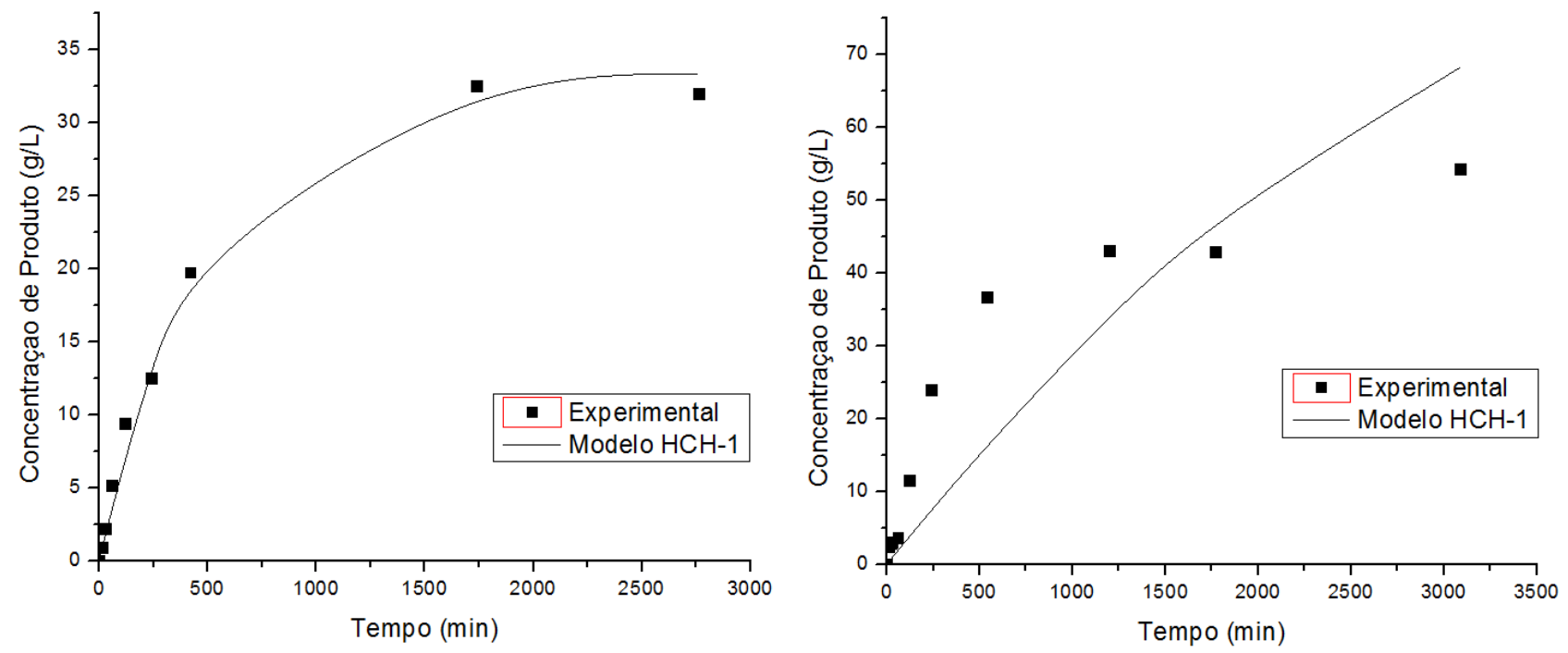

Figura 2: Gráficos de concentração de produto em função do tempo. À esquerda o gráfico relacionado ao conjunto 1 e a direita o gráfico relacionado ao conjunto 2 .

Analisando as curvas experimentais e simuladas mostradas na Figura 2, para o modelo HCH-1 de O`Dwyeret al 2007, pode-se perceber quepara o primeiro conjunto de dados há uma boa correspondência entre o modelo (linha contínua) e os dados experimentais (pontos), o que valida o modelo adotado. Já para o segundo conjunto de dados, vê-se que o ajuste obtido não foi totalmente satisfatório (embora haja certa coerência nos valores dos parâmetros obtidos, conforme a Tabela 3). Sugere-se, pois, o uso do modelo de catálise enzimática com contribuição do efeito de "jamming" de Xu e Ding, 2007, para essa condição de hidrólise. 


\section{9 a 22 de outubro de 2014 \\ Florianópolis/SC}

\section{CONCLUSÃO}

Do presente trabalho pode-se concluir que o modelo decatálise enzimática com contribuição do efeito de "jamming" de Xu eDing (2007) forneceu boa correspondência com os dados experimentais de Carvalho (2011), para os dois conjuntos de dados estudados. Já para o caso do modelo HCH-1 de O`Dwyer, et al. (2007), esse foi adequado para modelagem do conjunto de dados 1. Somando-se o estudo de Carvalho (2011) que aplicou os modelos de Michaelis Mentenpseudohomogêneo e heterogêneo com inibição e o modelo de Chrastil aos mesmos conjuntos de dados, têmsediversas aplicações bem sucedidas da modelagem matemática semi-mecanistica da hidrólise enzimática. Tal fatocorrobora a ideia da utilização desta classe de modelos simples, principalmente dentro da perspectiva de aplicação em engenharia de biorreatores. Suas equações, contendo poucos parâmetros, são baseadas em hipóteses que representam de forma simplificada a realidade, mas ainda assim se ajustam bem aos dados experimentais e podem ser utilizadas nodesign, otimização e scaleup dos reatores enzimáticos.

\section{REFERÊNCIAS BIBLIOGRÁFICAS}

ALMEIDA, C.L. Etanol Celulósico. Universidade Federal do Ceará - Programa de PósGraduação em Engenharia Agrícola. Seminários. Disponível em: <http://www.ppgea.ufc.br/arquivos_download/Etanol\%20Celulosico.pdf> Acesso em: 02 jul. 2013.

BUCKERIDGE, M.S; SANTOS, W.D;SOUZA, A.P. As rotas para o etanol celulósico no brasil. In CORTEZ, 1.a.b. bioetanol de cana-de-açúcar. p\&d para produtividade e sustentabilidade. São Paulo: Blucher, 2010. P. 365-380.

CARVALHO, M.L, Kinect Study of the Enzymatic Hydrolysis of Sugarcane Bagasse. São Carlos: Departamento de Engenharia Química, UFSCar, 2011. Dissertação (Mestrado).

GALBE, M. ZACCHI, G. Pretreatment of lignocellulosic Materials for Efficient Bioethanol Production.Advances in BiochemicalEngineering/biotechnology. Vol.108 p41-65 2007.

LORA, E.E.S.; CORAL, D.S.O; ROCHA, M.H. Rotas termoquímica e bioquímica para biocombustíveis: estado-da-arte, oportunidades e desafios para o Brasil. AGRENER GD 2008 - $7^{\circ}$ Congresso Internacional sobre Geração Distribuída e Energia no Meio Rural . Disponível em: <http://www.nipeunicamp.org.br/agrener/anais/2008/Artigos/23.pdf?3bc2dbeb0a7bf779fe39c865b70 b3b3f=0d57f4f84e4640be3c9224acbd738f5a> Acesso em: 10 maio de 2013.

MARQUES, F. O alvo é o bagaço. Pesquisa FAPESP, n.163, p.17-25, set. 2009.

NELLES, Oliver. Nonlinear Local Optimization. Ln: . Nonlinear System Identification: From Classical Approaches to Neural Networks and Fuzzy Models. $1^{\circ}$ ed.Springer, 2001. P. 79 112.

NELLES, Oliver. Nonlinear Global Optimization. Ln: . Nonlinear System Identification: From Classical Approaches to Neural Networks and Fuzzy Models. $1^{\text {o }}$ ed. Springer, 2001. P. 113 120. 
O`DWYER ET AL. Enzymatic hydrolysis of lime-pretreated corn stover and investigation os the HCH-1 Model: Inhibition pattern, degree of inhibition, validyos simplified HCH-1 Model. Bioresource Technology v.98 n.16 p 2969-2977, nov 2007.

OGEDA, T.L. PETRI, D.F.S. Hidrólise Enzimática de Biomassa. Química Nova. Vol 333, n.7 p1549-1558 jul. 2007.

SOUSA JR. ET AL. Recent trends in the modeling of celulose hydrolysis. Brazilian Journal of Chemical Engineering.V28 n.4, p545-564, out/dec 2011.

XU F. e DING, H. A new Kinect model for heterogeneous (or spatially confined) enzymatic catalysis: Contribution from the fractal and jamming (overcrowding) effects. Applied Catalysis A: General v.317 n.01 p.70-81, jan 2007 